\title{
J-Wave Elevation in the Inferior Leads Predicts Lethal Ventricular Arrhythmia Initiated by Premature Ventricular Contractions With Right Bundle Branch Block and Superior Axis
}

\author{
Yumi Munetsugu, MD; Mitsuharu Kawamura, MD; Toshihiko Gokan, MD; Ko Ogawa, MD; \\ Yuya Nakamura, MD; Akinori Ochi, MD; Koichiro Inokuchi, MD; Hiroyuki Ito, MD; \\ Tatsuya Onuki, MD; Youichi Kobayashi, MD; Toshiro Shinke, MD
}

\begin{abstract}
Background: Lethal ventricular arrhythmia (VA) can be initiated by idiopathic premature ventricular contractions (PVCs) originating from the left ventricular (LV) inferior wall. Furthermore, J-wave elevation in the inferior leads on ECG is sometimes associated with lethal VA. However, the relationship between these PVCs and J-wave elevation in patients with lethal VA is unclear, so we investigated it in the present study.
\end{abstract}

Methods and Results: We studied 32 consecutive patients who underwent radiofrequency (RF) ablation of idiopathic PVCs with right bundle branch block (RBBB) and superior axis. Thee PVCs were originating from the inferior wall of the LV. Lethal VA was defined as ventricular fibrillation (VF) or ventricular tachycardia (VT) with loss of consciousness (LOC). Among 32 patients, 3 had VF and 2 had VT with LOC. Other 27 had non-lethal VA. Baseline clinical characteristics were not significantly difference between lethal and non-lethal VA. The ratio of J-wave elevation in lethal VA was significantly higher as compared with non-lethal VA (100\% vs. $11.1 \%, P<0.0001)$. Furthermore, no patients with $\mathrm{J}$-wave elevation in the inferior leads had recurrence of lethal VA after RF ablation of the PVCs.

Conclusions: We speculate that J-wave elevation in the inferior leads might be a predictor of lethal VA initiated by PVCs with RBBB and superior axis. RF ablation of these PVCs was a useful method of treating lethal VA.

Key Words: J-wave elevation; Lethal ventricular arrhythmia; Premature ventricular contractions; Right bundle branch block

$\mathbf{P}$ remature ventricular contractions (PVCs) with a right bundle branch block (RBBB) and a superior axis in patients without structure heart disease are known as non-lethal ventricular arrhythmia (VA). Sometimes, ventricular fibrillation (VF) and ventricular tachycardia (VT) with loss of consciousness (LOC) can be initiated by PVCs with RBBB and superior axis, ${ }^{1}$ which originate from the left ventricular (LV) posterior fascicle (LPF), the LV posterior papillary muscles (PPM) and mitral annulus (MA). ${ }^{24}$ Among patients with idiopathic lethal VA, some show J-wave elevation in the inferior leads on ECG during sinus rhythm. J-wave elevation is commonly considered benign, although several reports were suggested an association of lethal VA with J-wave elevation in the inferior leads. ${ }^{1,5,6}$ However, the relationship between idiopathic PVCs with RBBB and superior axis and J-wave elevation in the inferior leads is unclear, so the aim of this study was to investigate this relationship in patients with lethal VA.

\begin{abstract}
Methods
Study Population and Definition

We enrolled 32 consecutive patients with documented idiopathic VA initiated by PVCs with RBBB and superior axis and only PVCs with RBBB and superior axis. Of them, 3 patients with VF were emergency hospitalized, and trigger PVCs were documented by ECG monitoring or the event record of the implantable cardioverter defibrillator (ICD). The morphology of trigger PVCs with RBBB and superior axis were recorded by 12-lead ECG. A total of 15 patients with VT were admitted to the hospital as an emergency, and all patients hoped for ablation of VT; the remaining 14 patients with PVC had symptoms and a PVC burden $>10 \%$ by 24-h Holter monitoring. Furthermore, all patients hoped for ablation of the PVCs.

All 32 patients had symptoms or prior events and 31 patients underwent radiofrequency (RF) ablation of idiopathic VA. None of the patients had identifiable structural
\end{abstract}

Received January 20, 2019; revised manuscript received June 3, 2019; accepted June 17, 2019; J-STAGE Advance Publication released online August 8, 2019 Time for primary review: 16 days

Division of Cardiology, Department of Medicine, Showa University School of Medicine, Tokyo, Japan

Mailing address: Mitsuharu Kawamura, MD, Division of Cardiology, Department of Medicine, Showa University School of Medicine, 1-5-8 Hatanaodai, Shinagawa-ku, Tokyo 142-8666, Japan. E-mail: mitsuhitoharu@yahoo.co.jp

ISSN-1346-9843 All rights are reserved to the Japanese Circulation Society. For permissions, please e-mail: cj@j-circ.or.jp 
heart disease as demonstrated by normal echocardiographic biventricular dimensions and function ( $\mathrm{LV}$ ejection fraction $(\mathrm{LVEF})>50 \%$ ), no detectable coronary artery disease on coronary angiography or exercise testing, and no known repolarization abnormalities. PVC/VT-induced cardiomyopathy was defined as an LVEF $<50 \%$ before ablation and EF normalized and clearly improved by $\geq 10 \%$ after 3 months. We excluded PVC/VT-induced cardiomyopathy from structural heart disease. QT interval corrected for heart rate (QTc) $<340 \mathrm{~ms}$ (short QT interval) or $>440 \mathrm{~ms}$ (long QT interval) at baseline were exclusion criteria. We defined the VA-QRS duration as QRS duration during VA (trigger PVC of VF, monomorphic VT and PVC). Patients with Brugada syndrome (BrS), defined as RBBB and ST-segment elevation $(\geq 0.2 \mathrm{mV})$ in V1-3 at baseline or following sodiumchannel blocker infusion were also excluded. In addition, patients with catecholaminergic arrhythmias, defined as arrhythmias during catecholamine infusion or exercise testing, were excluded. ${ }^{1}$

\section{Criterion of $\mathrm{J}$-Wave Elevation in the Inferior Leads}

The 12-lead ECG was recorded at a paper speed of $25 \mathrm{~mm} / \mathrm{s}$ with amplification of $10 \mathrm{~mm} / \mathrm{mV}$ in all patients. According to a previous study, ${ }^{1} \mathrm{~J}$-wave elevation was defined as an elevation of the QRS-ST junction (J-point) in at least 2 inferior leads (II, III and aVF). The J-wave elevation amplitude had to be at least $1 \mathrm{~mm}(0.1 \mathrm{mV})$ above the baseline level, either as QRS slurring (smooth transition from QRS to the ST-segment) or notching (positive $\mathrm{J}$ deflection inscribed on the $\mathrm{S}$ wave). ${ }^{1,5}$ All ECG were reviewed by at least 2 electrophysiologists.

\section{Classification of Groups}

The patients were categorized into 2 groups based on the presence or absence of lethal VA, which was defined as VF or VT with LOC, triggered by PVCs with RBBB and superior axis. Non-lethal VA was defined as only PVCs with $\mathrm{RBBB}$ and superior axis and/or VT without LOC initiated by PVCs with RBBB and superior axis.

\section{Definition of Origin of VA With RBBB and Superior Axis}

In the case of VF, we ablated the initiating PVCs. In the case of VT or only PVCs, we ablated the clinical VT or PVCs. The origin of the target VA was defined as the sites of the earliest ventricular activation or the established site of successful ablation. We used a 3D activation map and fluoroscopic images to clarify the anatomical image. The LPF site was defined by the LV posteroseptal region with intracardiac electrogram recordings showing high-frequency Purkinje potentials preceding the QRS onset during VA and sinus rhythm. The PPM site was defined by anatomical landmarks and a successful ablation site, located in the mid-inferior LV region and confirmed by intracardiac echocardiography (ICE) and fluoroscopic images. The MA site was defined by catheter tip recordings demonstrating a characteristic MA site (both atrial and ventricular electrogram signals) together with a successful ablation site established by $3 \mathrm{D}$ activation map and fluoroscopic images.

\section{Electrophysiological and RF Ablation Protocol}

Quadripolar electrode catheters were introduced into the femoral and subclavian veins and positioned in the right ventricular (RV) apex, coronary sinus and His bundle. If target PVCs/VT did not occur spontaneously, programmed ventricular stimulation was performed from the RV apex and right ventricular outflow tract (RVOT) using up to 3 extra-stimulations. Rapid burst pacing was also performed from 2 sites (RV apex and RVOT). A 3D mapping system (CARTO, Biosence Webster, Inc., Diamond Bar; CA, USA or EnSite NavX, St. Jude Medical, Inc., Saint Paul; MN, USA) was used for all patients. A voltage map of the LV was created during sinus rhythm, and voltage was defined as follows: $0.1-1.0 \mathrm{mV}$ as the border zone. If the origin of the PVCs was papillary muscle, ICE was used to ensure adequate catheter contact. Conventional mapping techniques including pace-mapping and activation mapping were used to identify the target site for RF ablation. If clinical VA occurred during the electrophysiological study, the ablation site was determined by the earliest activation site on $3 \mathrm{D}$ mapping. If there was no clinical VA during electrophysiological study, the ablation site was determined by pacemapping. RF ablation was performed using a $3.5-\mathrm{mm}$ open-irrigated-tip ablation catheter (Thermocool Celsius or Navistar, Biosence Webster Inc.). RF energy was delivered as $30 \mathrm{~W}$ with gradual titration to $50 \mathrm{~W}$. A maximum target temperature was $50^{\circ} \mathrm{C}$ and the target impedance drop was $10 \mathrm{ohm}$. Successful ablation was defined as the absence of any VA with the same morphology of the targeted VA after a 30-min waiting period after the final ablation and noninducibility of clinical VA. ${ }^{7}$ In patients with only PVCs, recurrence was defined as $\angle 80 \%$ reduction of the burden of PVCs on 24-h Holter recording. In other patients with VF or VT, recurrence was defined as the appearance of ablated VA on 24-h Holter recording. During the follow-up period of 24 months after ablation, a surface 12-lead ECG was recorded monthly and 24-h Holter recording was performed at several month intervals. If patients had any symptoms suggestive of recurrence of VA, such as palpitations or syncope, we performed 12-lead ECG and 24-h Holter recording.

\section{Statistical Analysis}

Continuous variables are expressed as mean $\pm \mathrm{SD}$ and compared using the Student $t$ test or analysis of variance for normally distributed variables. Fisher exact tests, when appropriate, were used to compare differences between categorical variables. Univariate analysis of variables was performed. $\mathrm{P}<0.05$ was considered statistically significant. The study was approved by the medical ethical review committees of Showa University School of Medicine. Written informed consent was given by all individuals.

\section{Results}

\section{Clinical Characteristics}

Table 1 shows the baseline clinical characteristics, ECG and electrophysiological characteristics of the patients with lethal VA (nos. 1-5) and non-lethal VA (nos. 6-32). The study population $(n=32)$ consisted of 22 males and 10 females (mean age $50.4 \pm 16.5$ years) with symptomatic idiopathic VA with RBBB and superior axis. In the lethal VA group, 3 patients (nos. 2, 3 and 5) had an episode of VF and the other 2 patients (nos. 1 and 4) had an episode of monomorphic VT with LOC. In the non-lethal VA group, 13 patients had an episode of monomorphic VT without LOC and the other 14 patients had an episode of only PVCs. All cases of VF and VT were initiated by PVCs with RBBB and superior axis. Among the 32 patients, 8 patients had J-wave elevation in the inferior leads. In the lethal VA group, all 5 patients had J-wave elevation. In the non-lethal VA group, only 3 patients had J-wave elevation (VT: 1 patient, PVC: 


\begin{tabular}{|c|c|c|c|c|c|c|c|c|c|c|c|}
\hline $\begin{array}{l}\text { Patient } \\
\text { no. }\end{array}$ & $\begin{array}{c}\text { Lethal or } \\
\text { non-lethal } \\
\text { VA }\end{array}$ & $\begin{array}{c}\text { Age } \\
\text { (years) }\end{array}$ & Sex & $\underset{\text { VA }}{\text { Clinical }}$ & Symptoms & $\begin{array}{l}\text { J-wave } \\
\text { elevation }\end{array}$ & $\begin{array}{l}\text { LVEF } \\
(\%)\end{array}$ & $\begin{array}{c}\text { Origin of } \\
\text { target VA } \\
\text { and RF site }\end{array}$ & $\begin{array}{l}\text { VA-QRS } \\
\text { duration } \\
\text { (ms) }\end{array}$ & $\begin{array}{c}\text { PVC } \\
\text { coupling } \\
\text { interval (ms) }\end{array}$ & $\begin{array}{c}\text { ICD } \\
\text { implantation }\end{array}$ \\
\hline 1 & $\mathrm{~L}$ & 77 & M & Mono-VT & LOC & + & 67 & $\begin{array}{l}\text { Posterior } \\
\text { fascicle }\end{array}$ & 180 & 498 & - \\
\hline 2 & L & 47 & M & VF & LOC & + & 54 & $\begin{array}{l}\text { Posterior } \\
\text { papillary }\end{array}$ & 171 & 481 & + \\
\hline 3 & L & 31 & M & VF & LOC & + & 65 & Mitral annulus & 160 & 398 & + \\
\hline 4 & L & 41 & $\mathrm{~F}$ & Mono-VT & LOC & + & 55 & $\begin{array}{l}\text { Posterior } \\
\text { papillary }\end{array}$ & 150 & 397 & - \\
\hline 5 & L & 13 & M & VF & LOC & + & 61 & Undefined & 140 & 330 & + \\
\hline 6 & $\mathrm{NL}$ & 64 & $\mathrm{~F}$ & PVC & None & - & 51 & $\begin{array}{l}\text { Posterior } \\
\text { papillary }\end{array}$ & 128 & 486 & - \\
\hline 7 & $\mathrm{NL}$ & 76 & M & PVC & None & - & 58 & $\begin{array}{l}\text { Posterior } \\
\text { papillary }\end{array}$ & 140 & 500 & - \\
\hline 8 & $\mathrm{NL}$ & 67 & $\mathrm{~F}$ & PVC & None & - & 54 & $\begin{array}{l}\text { Posterior } \\
\text { papillary }\end{array}$ & 146 & 412 & - \\
\hline 9 & $\mathrm{NL}$ & 72 & M & PVC & None & + & 71 & $\begin{array}{l}\text { Posterior } \\
\text { papillary }\end{array}$ & 163 & 558 & - \\
\hline 10 & $\mathrm{NL}$ & 40 & $\mathrm{~F}$ & PVC & None & - & 59 & $\begin{array}{l}\text { Posterior } \\
\text { papillary }\end{array}$ & 146 & 532 & - \\
\hline 11 & $\mathrm{NL}$ & 41 & M & Mono-VT & None & + & 52 & $\begin{array}{l}\text { Posterior } \\
\text { fascicle }\end{array}$ & NA & NA & - \\
\hline 12 & NL & 35 & M & Mono-VT & None & - & 57 & $\begin{array}{l}\text { Posterior } \\
\text { fascicle }\end{array}$ & 140 & NA & - \\
\hline 13 & $\mathrm{NL}$ & 71 & M & PVC & None & - & 68 & Mitral annulus & 160 & 740 & - \\
\hline 14 & NL & 55 & M & Mono-VT & None & - & 62 & $\begin{array}{l}\text { Posterior } \\
\text { fascicle }\end{array}$ & 129 & 478 & - \\
\hline 15 & NL & 66 & M & PVC & None & - & 55 & $\begin{array}{l}\text { Posterior } \\
\text { papillary }\end{array}$ & 150 & 458 & - \\
\hline 16 & $\mathrm{NL}$ & 68 & M & PVC & None & - & 65 & $\begin{array}{l}\text { Posterior } \\
\text { papillary }\end{array}$ & 159 & 487 & - \\
\hline 17 & NL & 41 & M & Mono-VT & None & - & NA & $\begin{array}{l}\text { Posterior } \\
\text { fascicle }\end{array}$ & 136 & 502 & - \\
\hline 18 & NL & 70 & $\mathrm{~F}$ & PVC & None & - & 68 & $\begin{array}{l}\text { Posterior } \\
\text { papillary }\end{array}$ & 160 & 479 & - \\
\hline 19 & $\mathrm{NL}$ & 36 & M & Mono-VT & None & - & 36 & $\begin{array}{l}\text { Posterior } \\
\text { fascicle }\end{array}$ & 123 & 496 & - \\
\hline 20 & NL & 57 & $\mathrm{~F}$ & Mono-VT & None & - & 80 & $\begin{array}{l}\text { Posterior } \\
\text { fascicle }\end{array}$ & 120 & 406 & - \\
\hline 21 & NL & 51 & $F$ & Mono-VT & None & - & 60 & $\begin{array}{l}\text { Posterior } \\
\text { papillary }\end{array}$ & 152 & 712 & - \\
\hline 22 & NL & 44 & M & Mono-VT & None & - & 56 & $\begin{array}{l}\text { Posterior } \\
\text { fascicle }\end{array}$ & 135 & 411 & - \\
\hline 23 & NL & 38 & M & Mono-VT & None & - & 54 & $\begin{array}{l}\text { Posterior } \\
\text { fascicle }\end{array}$ & 149 & 443 & - \\
\hline 24 & NL & 37 & $F$ & Mono-VT & None & - & 60 & $\begin{array}{l}\text { Posterior } \\
\text { fascicle }\end{array}$ & 91 & 565 & + \\
\hline 25 & NL & 63 & $M$ & PVC & None & - & 66 & $\begin{array}{l}\text { Posterior } \\
\text { papillary }\end{array}$ & 140 & 452 & - \\
\hline 26 & NL & 24 & $\mathrm{~F}$ & PVC & None & + & 65 & $\begin{array}{l}\text { Posterior } \\
\text { papillary }\end{array}$ & 126 & 456 & - \\
\hline 27 & NL & 25 & $\mathrm{~F}$ & Mono-VT & None & - & 48 & $\begin{array}{l}\text { Posterior } \\
\text { fascicle }\end{array}$ & 123 & 455 & - \\
\hline 28 & NL & 54 & $M$ & PVC & None & - & 73 & $\begin{array}{l}\text { Posterior } \\
\text { papillary }\end{array}$ & 156 & 565 & - \\
\hline 29 & NL & 40 & M & Mono-VT & None & - & 67 & $\begin{array}{l}\text { Posterior } \\
\text { fascicle }\end{array}$ & 105 & 443 & - \\
\hline 30 & NL & 50 & M & Mono-VT & None & - & 60 & $\begin{array}{l}\text { Posterior } \\
\text { papillary }\end{array}$ & 127 & 564 & - \\
\hline 31 & NL & 63 & $M$ & PVC & None & - & 62 & $\begin{array}{l}\text { Posterior } \\
\text { papillary }\end{array}$ & 150 & 416 & - \\
\hline 32 & NL & 57 & M & PVC & None & - & 59 & $\begin{array}{l}\text { Posterior } \\
\text { papillary }\end{array}$ & 135 & 458 & - \\
\hline
\end{tabular}

L, lethal; LOC, loss of consciousness; LVEF, left ventricular ejection fraction; Mono-VT, monomorphic VT; NL, non-lethal; RF, radiofrequency; VA, Ventricular Arrhythmia; VA-QRS duration, QRS duration during VA (trigger PVC of VF; mono-VT and PVC). 


\begin{tabular}{lccc|}
\hline \multicolumn{1}{l}{ Table 2. Comparison of Lethal and Non-Lethal VA Patients } & & \\
& $\begin{array}{c}\text { Lethal VA } \\
(\mathbf{n = 5 )}\end{array}$ & $\begin{array}{c}\text { Non-lethal VA } \\
(\mathbf{n = 2 7})\end{array}$ & P value \\
Age (years) & $41.8 \pm 23.5$ & $52.0 \pm 15.0$ & 0.3 \\
Sex: male (\%) & $4(80 \%)$ & $18(66.6 \%)$ & 0.5 \\
LVEF (\%) & $60.4 \pm 5.8$ & $61.1 \pm 7.3$ & 0.9 \\
Past history & & & \\
Hypertension & $1(20 \%)$ & $7(26 \%)$ & 0.7 \\
Hyperlipidemia & $1(20 \%)$ & $1(0.3 \%)$ & 0.1 \\
Diabetes & $0(0 \%)$ & $1(0.3 \%)$ & 0.6 \\
CKD & $0(0 \%)$ & $0(0 \%)$ & \\
Antiarrhythmic drugs & & & 0.5 \\
Class Ic & $0(0 \%)$ & $2(0.7 \%)$ & 0.2 \\
Class II & $0(0 \%)$ & $6(22 \%)$ & 0.2 \\
Class III & $0(0 \%)$ & $6(22 \%)$ & 0.5 \\
Class IV & $2(40 \%)$ & $7(26 \%)$ & $<0.0001$ \\
J-point elevation (\%) & $5(100 \%)$ & $3(11.1 \%)$ & 0.019 \\
VA-QRS duration (ms) & $160 \pm 15.9$ & $138 \pm 17.4$ & 0.06 \\
PVC coupling interval (ms) & $420 \pm 68$ & $498 \pm 83$ & 0.01 \\
PVC burden (\%) & $5 \pm 10$ & $16 \pm 8$ & $N$ \\
Successful ablation & $4(100 \%)$ & $27(100 \%)$ & 0.29 \\
Recurrence & $0(0 \%)$ & $6(22 \%)$ & 0.003 \\
ICD & $3(60 \%)$ & $1(3.7 \%)$ & \\
\hline
\end{tabular}

CKD, chronic kidney disease; ICD, implantable cardioverter defibrillator; PVC, premature ventricular contraction. Other abbreviations as in Table 1.

2 patients). In the lethal VA group, initiating PVCs originated from the LPF $(n=1)$, PPM $(n=2)$ and MA $(n=1) ; 1$ patient did not undergo RF ablation because of young age. However, a subcutaneous ICD was inserted to treat the VF. In the non-lethal VA group, initiating PVCs originated from the LPF $(n=11)$, PPM $(n=15)$, and MA $(n=1)$.

\section{Comparison of Clinical Characteristics Between Lethal and Non-lethal VA}

Table 2 compares the clinical characteristics of the 2 VA groups. There were no significant differences in age, sex, LVEF or PVC coupling interval between groups: age (years): $41.8 \pm 23.5$ vs. $52.0 \pm 15.0$; sex (male); $80.0 \%$ vs. $66.6 \%$; LVEF (\%); $60.4 \pm 5.8$ vs. $61.1 \pm 7.3$; PVC coupling interval (ms); $410 \pm 86$ vs. $498 \pm 83$,). As for medications, 1 patient with trigger PVCs of VF was resistant to bepridil and some patients with VT were resistant to amiodarone, sotalol, verapamil and $\beta$-blockers. The average burden of PVCs per day was $16 \%$. Some patients with PVCs were resistant to amiodarone, verapamil, $\beta$-blockers, propafenon or flecainide. The VA-QRS duration in the lethal VA group was significantly wider than in the non-lethal VA group $(160 \pm 15.9$ vs. $138 \pm 17.4 \mathrm{~ms}, \mathrm{P}=0.019)$. The average cycle length $(\mathrm{CL})$ of VTs in lethal VA was significantly shorter than in non-lethal VA $(260 \pm 14$ vs. $315 \pm 25 \mathrm{~ms}, \mathrm{P}=0.04)$. All patients in the lethal VA group had J-wave elevation in the inferior leads, whereas only 3 patients in the non-lethal VA group had J-wave elevation. The ratio of J-wave elevation in the lethal VA group was significantly higher than in the non-lethal VA group $(100 \%$ vs. $11.1 \%, \mathrm{P}<0.0001)$. Among the 32 patients, 31 underwent RF ablation of VA, and all had acute success; 1 young patient did not undergo RF ablation. The recurrence ratio of target VA was not significantly different between the lethal VA and non-lethal VA groups ( $0 \%$ vs. $22 \%, P=0.17)$, and all 4 patients with lethal VA had no recurrence. The ratio of ICD implantation was significantly higher in the lethal VA group than in the non-lethal VA group $(60 \%$ vs. $3.7 \%, \mathrm{P}=0.003)$. No complications occurred in our study. Among the 8 patients with $\mathrm{J}$-wave elevation, there were no significant differences in clinical characteristics (age, sex, LVEF, PVC coupling interval, VA-QRS duration, recurrence ratio and ratio of ICD implantation) between the lethal VA group and non-lethal VA group.

\section{Correlation Between Location of J-Wave Elevation and Arrhythmia Origin}

Figure 1 shows the 12-lead ECGs of 8 patients with J-wave elevation (nos. 1-5, 9, 11 and 26). The ECGs in patients 1, 2, 4, 5, 9 and 26 showed a notching pattern and in patients 3 and 11 it showed a slurring pattern. All 8 patients showed $\mathrm{J}$-wave elevation only in the inferior leads. In all 5 patients in the lethal VA group, initiating PVCs originated from the LPF, PPM and MA. In the other 3 patients in the nonlethal VA group, initiating PVCs originated from LPF and PPM. In both groups, the initiating PVCs originated from the LV inferior wall, which suggested a correlation between the location of J-wave elevation and PVC origin.

\section{Data Collected During Catheter Ablation}

The outcomes of catheter ablation are shown in Table 3 (lethal VA vs. non-lethal VA group). At the successful ablation site, 1 patient in the lethal VA group $(25 \%)$ had a Purkinje potential, while 11 patients in non-lethal VA group $(41 \%)$ had a Purkinje potential. There was no significant difference between groups. None of the patients had abnormal potentials or low voltage areas.

Typical Case Report: Patient No. 3

Figure 2 shows a typical case. A 32-year-old man with resuscitated idiopathic VF received an ICD during his 1st 


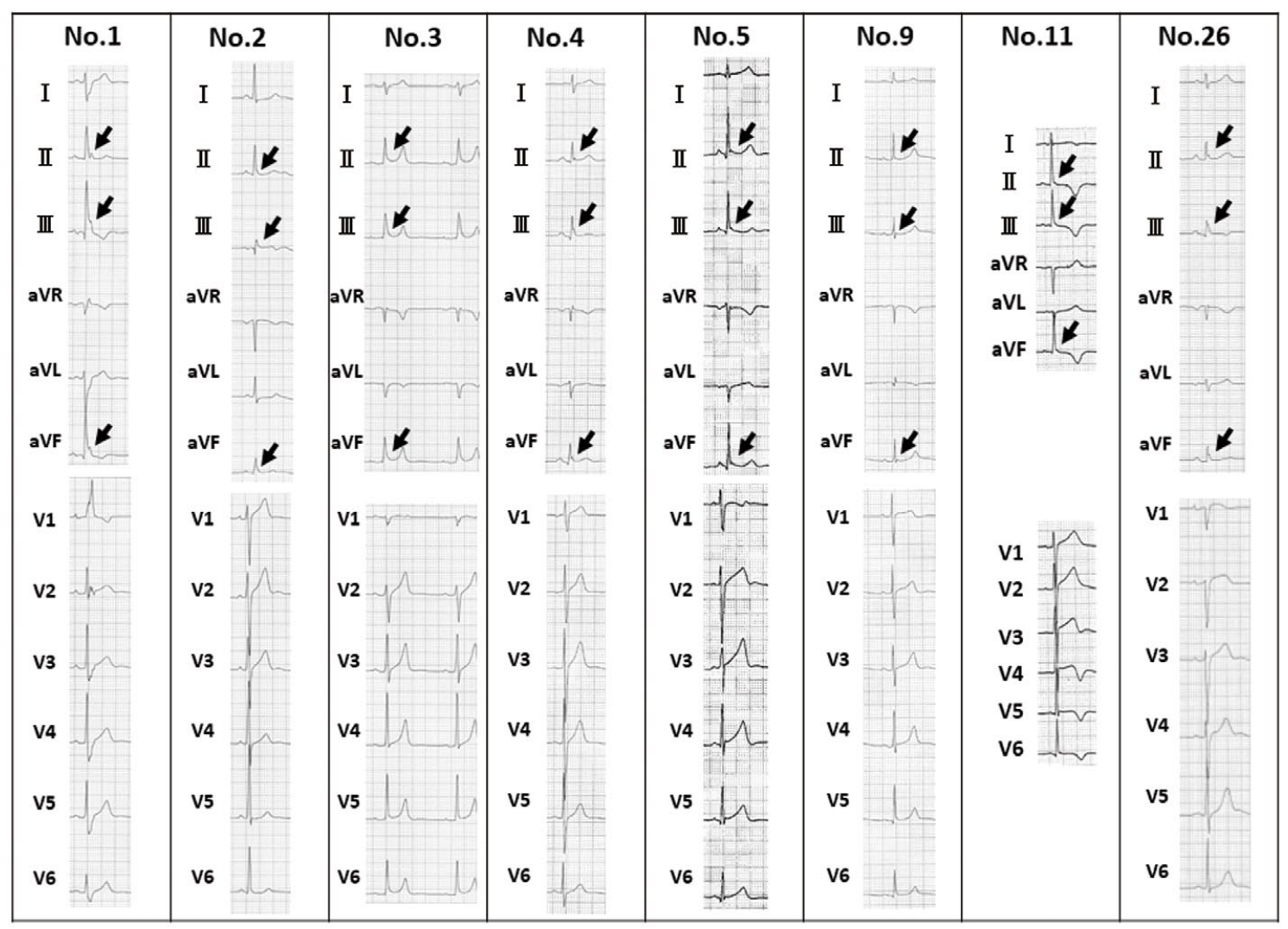

Figure 1. Morphology of J-wave elevation on 12-lead ECGs of 8 patients (nos.1-5, 9, 11 and 26). Patients 1, 2, 4, 5, 9 and 26 show a notching pattern (arrow), whereas nos. 3 and 11 show a slurring pattern (arrow). All 8 patients show J-wave elevation only in the inferior leads (II, III, aVF). The morphology of the J-wave elevation did not differ between lethal and non-lethal ventricular arrhythmia.

hospitalization and thereafter bepridil was effective in preventing recurrence of VF. However, 7 months later, he had an electrical storm and was admitted to hospital. The ICD record revealed 12 shock therapies for VF. In all episodes, VF was initiated by PVCs which all had same morphology (Figure 2A): RBBB and superior axis. These PVCs were originating from the inferoseptal LV. J-wave elevation during sinus rhythm was shown in the ECG inferior leads. The initiating PVCs originated from the LV inferior wall, possibly the same area showing the J-wave elevation (Figure 2B). Figure 2C shows the voltage mapping of the LV using the CARTO system during sinus rhythm. The initiating PVCs did not appear during the electrophysiological study, so we performed pace-mapping in the LV to identify the target site for RF ablation. After RF ablation, VF initiated by PVCs was not detected by ICD report for 4 years.

\section{Intracardiac ECG of Patients With J-Wave Elevation}

Figure 3 shows the bipolar and unipolar ICE at the successful ablation site. We show the ICE of 5 patients with J-wave elevation, both in sinus rhythm and ectopy. The trigger PVC of $\mathrm{VF}$ during ablation in patient 3 could not be recorded. In the lethal VA group, patient no. 1 had a Purkinje potential at the successful ablation site. In the non-lethal VA group, patient no. 11 had a Purkinje potential at the successful ablation site.

\section{Discussion}

\section{Main Findings}

The main findings of our study are as follows. (1) The ratio of J-wave elevation in the lethal VA group was significantly higher than in the non-lethal VA group $(100 \%$ vs. $11.1 \%, \mathrm{P}<0.0001)$. (2) The initiating PVCs of lethal VA showed RBBB and a superior axis, which originated from the LPF, PPM and MA in the inferior LV wall; that is, originating from the same area showing J-wave elevation in the ECG inferior leads. (3) RF ablation of the initiating PVCs in patients with lethal VA was curative and none of the patients had recurrence of lethal VA during the followup period. Therefore, RF ablation for lethal VA with J-wave elevation might be an effective treatment.

\section{Comparison of Monomorphic VT With and Without LOC}

VT patients (nos. 1 and 4) with LOC were documented with only monomorphic VT, so we considered that the shorter $\mathrm{CL}$ and wider QRS duration induced VT with LOC in the lethal VA group.

A previous report showed the relationship between the early repolarization (ER) pattern and E/e' in soccer players and patients' backgrounds might be strongly related to LOC. ${ }^{8}$ In our study, there were no significant differences in the baseline characteristics of 2 groups (lethal VA and non- 


\begin{tabular}{|c|c|c|c|}
\hline & $\begin{array}{l}\text { Lethal VA } \\
\qquad(n=4)\end{array}$ & $\begin{array}{l}\text { Non-lethal VA } \\
\quad(n=27)\end{array}$ & $P$ value \\
\hline Purkinje potential & $1(25 \%)$ & $11(41 \%)$ & 0.5 \\
\hline Abnormal potential & 0 & 0 & NS \\
\hline Low-voltage area $(<1.5 \mathrm{mV})$ & 0 & 0 & NS \\
\hline \multicolumn{4}{|c|}{ Ablation of trigger PVC of VF or PVC } \\
\hline & $\begin{array}{l}\text { Trigger PVC of } \\
\text { VF }(n=2)\end{array}$ & $\begin{array}{l}\text { PVC } \\
(n=14)\end{array}$ & $P$ value \\
\hline \multicolumn{4}{|l|}{ Mapping } \\
\hline Activation map: earliest (ms) & 42 & $39 \pm 10$ & 0.6 \\
\hline Pace-mapping score (\%) & 100 & $96 \pm 5$ & 0.6 \\
\hline \multicolumn{4}{|l|}{ Ablation site } \\
\hline Posterior papillary & $1(50 \%)$ & $13(93 \%)$ & 0.08 \\
\hline Mitral annulus & $1(50 \%)$ & $1(7 \%)$ & 0.08 \\
\hline \multicolumn{4}{|l|}{ Ablation of Monomorphic VT } \\
\hline & $\begin{array}{l}\text { Monomorphic } \\
\text { VT }(n=2)\end{array}$ & $\begin{array}{l}\text { Monomorphic } \\
\text { VT }(n=13)\end{array}$ & $P$ value \\
\hline VT induction & $2(100 \%)$ & $7(54 \%)$ & 0.2 \\
\hline \multicolumn{4}{|l|}{ Mapping } \\
\hline Activation map: earliest (ms) & $35 \pm 7$ & $31 \pm 6$ & 0.5 \\
\hline Pace-mapping score (\%) & & $97 \pm 3$ & \\
\hline \multicolumn{4}{|l|}{ Ablation site } \\
\hline Posterior papillary & $1(50 \%)$ & $2(15 \%)$ & 0.2 \\
\hline Posterior fascicle & $1(50 \%)$ & $11(85 \%)$ & 0.2 \\
\hline
\end{tabular}

VF, ventricular fibrillation; VT, ventricular tachycardia. Other abbreviations as in Tables 1,2.

lethal VA). However, we did not investigate a parameter of LV diastolic function for all patients and we could not compare it in detail. Therefore, further studies are required to be certain about lethal VA and LV diastolic function.

\section{Origin of Initiating PVCs of Idiopathic VA}

Idiopathic PVCs are considered non-lethal VA, although in a previous study, PVCs originating from the RVOT was recognized as a potential trigger for idiopathic $\mathrm{VF} /$ polymorphic VT (PMVT).9,10 Another study also showed that idiopathic VF/PMVT was initiated by PVCs originating from a papillary muscle. ${ }^{11}$ Haïssaguerre et $\mathrm{al}^{\mathbf{9}}$ described the ablation of idiopathic VF initiated by PVCs with short coupling intervals. Those initiating PVCs were either in the peripheral Purkinje network system of both ventricles (20 patients) or the RVOT (4 patients). Furthermore, the inferolateral border of the RV or the Purkinje fibers of the moderator band areas have been targeted for idiopathic VF ablation.12,13 These intriguing findings suggest that the peripheral Purkinje network system of both ventricles might be involved in the mechanism of initiating VF by PVCs originating from the inferior LV wall. In our study, the initiating PVCs of lethal VA originated from the LPF, PPM and MA in the inferior LV wall; therefore, we speculate the peripheral Purkinje network system is associated with initiating lethal VA by PVCs originating from the inferior LV wall. However, in the non-lethal VA group, PVCs originating from the inferior LV wall (i.e., LPF, PPM and MA) did not induce lethal VA, and $\mathrm{J}$-wave elevation in the inferior leads was not found in most of these patients.

\section{Association With J-Wave Elevation and the Initiating PVCs of Lethal VA}

$\mathrm{J}$-wave elevation is a common ECG finding and considered an innocent sign among healthy young populations. ${ }^{\mathbf{1 4}}$ However, recent study showed that inferolateral J-wave elevation is not always benign and sometimes associated with idiopathic VF.1,15 Antzelevitch et a ${ }^{\mathbf{1 6}}$ proposed dividing the J-wave elevation syndrome into 4 subtypes (ER syndrome (ERS) types $1-4$ ). Type 2 predominantly displays a J-wave elevation pattern in the inferior leads and is associated with a higher level of risk of death from cardiac causes. In our study, all initiating PVCs and clinical PVCs with idiopathic VA had the morphology of RBBB and a superior axis. The originating site of these PVCs was identified as the inferior $\mathrm{LV}$ wall by $3 \mathrm{D}$ mapping system and ICE. Therefore, in the lethal VA group, the ratio of patients with J-wave elevation in the inferior leads was significantly higher than in the non-lethal VA group. From these results, we speculate that $\mathrm{J}$-wave elevation in the inferior leads represents a moderately arrhythmogenic substrate in the inferior wall. PVCs originating from the same area facilitate this arrhythmogenic substrate. Previous studies described some reasons for it. First, J-wave elevation in the inferior leads sometimes shows in healthy control patients, but is more common in patients with idiopathic VF. Second, the initiating PVCs of VF typically show a superior axis, which suggests these PVCs originate from the inferior wall., $, 17,18$ Therefore, these PVCs originate from the Purkinje fibers network.9,18 Third, PVCs originating from the Purkinje fibers network have a very short coupling interval, ${ }^{18}$ which suggests an abnormally short refractory period exists in the originating area of these PVCs. In our study, the initiating PVCs of lethal VA originated from the inferior LV wall, as in the previous studies. How- 
(A) Initiation of VF recorded by ICD

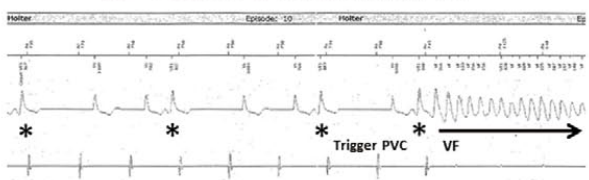

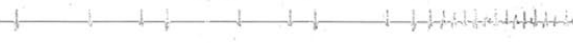

(B) 12 leads ECG : Initiating VF by monomorphic PVC
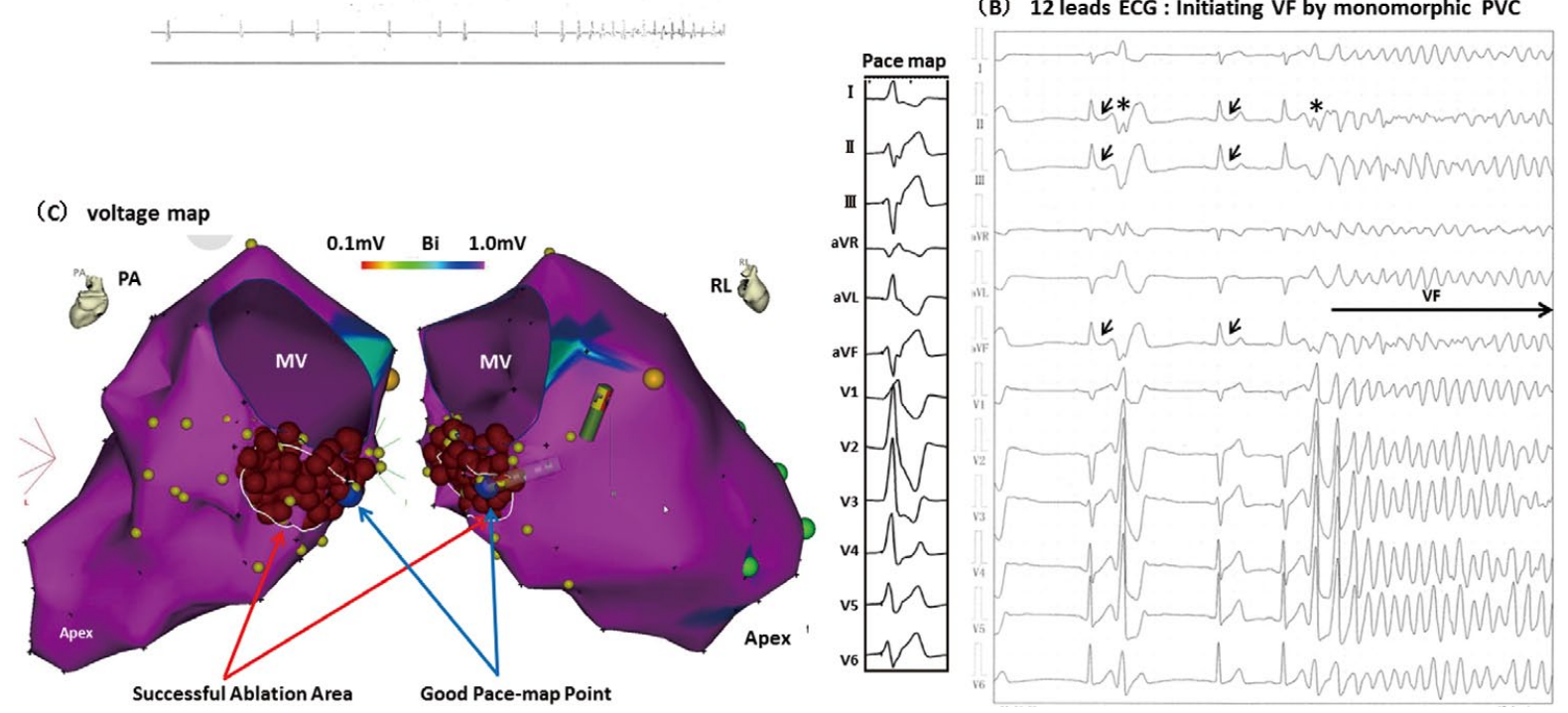

Figure 2. Typical case of the lethal ventricular arrhythmia in patient no. 3. (A) ICD report of shock therapies for ventricular fibrillation (VF). All episodes of VF were initiated by premature ventricular contractions (PVCs) ${ }^{*}$ ) and these PVCs had the same morphology. (B) Initiating PVCs $\left(^{*}\right)$ of VF show right bundle branch block (RBBB) and a superior axis, originating from the left ventricular (LV) inferior wall. J-wave elevation (arrow) is shown in the inferior leads (II, III, aVF). (C) 3D voltage map of the LV. The blue dot in the LV inferior wall represents the good pace-mapping point; radiofrequency (RF) ablation was performed in the LV inferior area (red dots reflect RF ablation lesions).

ever, initiating PVCs originating from the Purkinje fibers network occurred in only 1 patient in the lethal VA group. In the other 3 patients, the PVCs originated from the PPM or MA. Therefore, we speculate a different mechanism of lethal VA from those in previous studies. In our study, the originating site of the PVCs was consistent with the location of the J-wave elevation in the inferior leads. The arrhythmogenic substrate represented by J-wave elevation in the inferior leads on ECG was located in the same inferior LV wall; therefore, the PVCs originating from same area with J-wave elevation might be facilitated by the arrhythmogenic substrate. It might be more easily associated with the development of lethal arrhythmias. In a previous study, arrhythmic recurrence was more frequent in patients with $\mathrm{J}$-wave elevation as compared with those without such elevation. ${ }^{1}$ In our study, patient 3 was similar to these previous reports. ${ }^{15}$ He had an electrical storm after ICD implantation. Cilostazol was ineffective and bepridil could not control the VA during follow-up. RF ablation of lethal VA could be another modality in the management of VF in patients who fail antiarrhythmic therapy.

\section{Mechanism of Lethal VA in Patients With J-Wave Elevation}

The electrophysiological mechanisms responsible for J-wave elevation remain unclear, although 2 hypotheses based on clinical and experimental evidence suggest anomalies in delayed depolarization or ER. Antzelevitch et al proposed the mechanism of J-wave and ST-segment elevation as ER, and patients with BrS (ERS type 4) show marked changes in the right precordial leads (V1-3) and large dispersion of repolarization that allows phase II reentry polymorphic ventricular arrhythmia to occur even in the absence of triggering extra systoles. ${ }^{16,19}$ Nam et al ${ }^{20}$ proposed the VF episodes in patients with ERS type 3 were commonly initiated by PVCs with a short-long-short sequence. The initiating PVCs of VF in patients with ERS had a significantly shorter

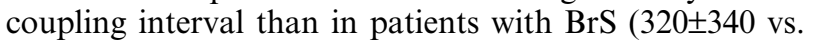
$350 \pm 404 \mathrm{~ms})$. The initiating PVCs were relatively shortcoupled, and consistent with a phase 11 re-entrant mechanism. These ECG changes portend the development of phase 11 reentry and suggest enhanced spatial dispersion of repolarization, which can give rise to a highly arrhythmogenic substrate. However, our study had some differences from previous studies. First, sodium-channel blockers had no effect on J-wave elevation, a response that favors a different mechanism of initiating VF from that of BrS. Second, in our study, the coupling interval of PVCs was longer (mean $443 \pm 53 \mathrm{~ms}$ ) as compared with previous studies of $\mathrm{BrS}$ and ERS type 3 . Nakagawa et $\mathrm{al}^{\mathbf{2 1}}$ proposed a possible association of false tendon (FT) with J-wave, and that the mechanism of J-wave elevation was delayed depolarization. The incidence of J-waves was significantly higher in patients with FT $(64 \%)$ than in patients without FT $(19 \%)(\mathrm{P}<0.001)$. The location of the J-wave depended on the varied shape and location of FT. The tissue inside the FT may have abnormal properties such as slower conduction of electrical activ- 


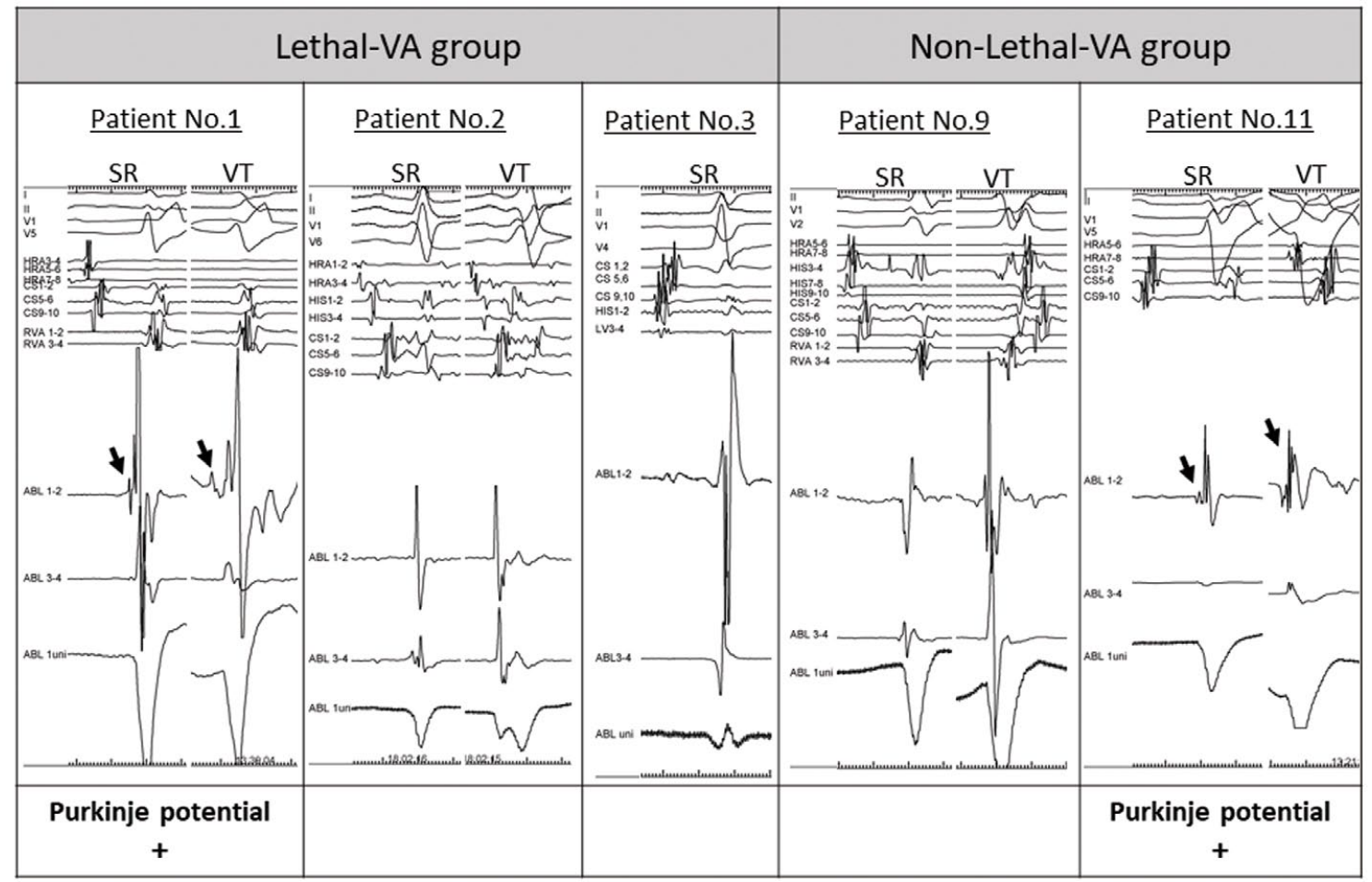

Figure 3. Bipolar and unipolar intracardiac electrocardiograms at the successful ablation site of 5 patients with J-wave elevation, both in sinus rhythm and ectopy. Patient no. 3 did not have the trigger premature ventricular contraction (PVC) of ventricular fibrillation (VF) recorded during ablation. In the lethal ventricular arrhythmia (VA) group, patient no. 1 had a Purkinje potential at the successful ablation site. In the non-lethal VA group, patient no. 11 had a Purkinje potential at the successful ablation site.

ity than the tissue of the normal His bundle or Purkinje fibers. This slow conduction may be anatomically directed inferolaterally and appear as delayed activity in the terminal portion of the QRS complex in the inferior or lateral leads of the surface ECG. In our study, the patient 3 showed FT on ICE during ablation, so the mechanism of J-wave elevation in the inferior leads might be delayed depolarization. In that patient, the PVCs originating from the same area with J-wave elevation could give rise to a highly arrhythmogenic substrate. Therefore, just only single- and longer coupled PVCs could be initiate VF. It might acutely increase the vulnerability of the ventricle and might promote the displacement of depolarization or repolarization before VF episodes.

\section{Study Limitations}

First, the small number of patients, because of the rarity of cases of idiopathic VF among patients with J-wave elevation in the inferior leads. Thus, the results should be interpreted with caution. Second, this study was a retrospective analysis, so the association between J-wave elevation in the inferior leads and lethal VA initiated by PVCs with RBBB and a superior axis cannot be quantified because our study selected only those patients who survived and were able to have their episodes documented in the hospital. Third, we could not completely define the mechanism of VF initiated by PVCs with RBBB and a superior axis. Therefore, further prospective studies are required to confirm the relationship between $\mathrm{J}$-waves in the inferior leads and the mechanism of lethal VA. Finally, in our study, 31 patients underwent ablation because of symptomatic VA or PVC burden $>10 \%$. Because of this selection bias and the small number of patients enrolled, the percent of lethal VA with RBBB and a superior axis was much higher. In other words, patients with VF or VT and LOC are more likely to be hospitalized and more likely to be referred for RF ablation while patients with monomorphic VT without LOC are more likely to be treated conservatively as outpatients. However, we believe that this study is an adequate evaluation because there was a significant difference between the lethal VA and non-lethal VA groups.

\section{Conclusions}

J-wave elevation in inferior leads was associated with lethal VA triggered by idiopathic PVCs with RBBB and a superior axis. We consider that J-wave elevation in the ECG inferior leads might be a predictor of lethal VA initiated by such PVCs. RF ablation of lethal VA triggered by PVCs could be another potential treatment modality in the management of lethal VA in patients with failed antiarrhythmic therapy.

\section{Acknowledgment}

Part of this work was accomplished at the Division of Cardiology, Department of Medicine, Showa University School of Medicine.

\section{Name of Grant}

None. 


\section{References}

1. Haïssaguerre M, Derval N, Sacher F, Jesel L, Deisenhofer I, de Roy L, et al. Sudden cardiac arrest associated with early repolarization. N Engl J Med 2008; 358: 2016-2023.

2. Doppalapudi H, Yamada T, McElderry HT, Plumb VJ, Epstein AE, Kay GN. Ventricular tachycardia originating from the posterior papillary muscle in the left ventricle: A novel clinical syndrome. Circ Arrhythmia Electrophysiol 2008; 1: 23-29.

3. Good E, Desjardins B, Jongnarangsin K, Oral H, Chugh A, Ebinger $\mathrm{M}$, et al. Ventricular arrhythmias originating from a papillary muscle in patients without prior infarction: A comparison with fascicular arrhythmias. Heart Rhythm 2008; 5: 15301537.

4. Yamada T, McElderry HT, Okada T, Murakami Y, Doppalapudi $\mathrm{H}$, Yoshida N, et al. Idiopathic focal ventricular arrhythmias originating from the anterior papillary muscle in the left ventricle. J Cardiovasc Electrophysiol 2009; 20: 866-872.

5. Rosso R, Kogan E, Belhassen B, Rozovski U, Scheinman MM, Zeltser D, et al. J-point elevation in survivors of primary ventricular fibrillation and matched control subjects: Incidence and clinical significance. J Am Coll Cardiol 2008; 52: 1231-1238.

6. Sacher F, Lim H, Haïssaguerre H. Sudden cardiac death associated with $\mathbf{J}$ wave elevation in the inferolateral leads: Insights from a multicenter registry. J Electrocardiol 2013; 46: 456-460.

7. Santoro F, Di Biase L, Hranitzky P, Sanchez JE, Santangeli P, Perini AP, et al. Ventricular fibrillation triggered by PVCs from papillary muscles: Clinical features and ablation. J Cardiovasc Electrophysiol 2014; 25: 1158-1164.

8. Wilhelm M, Brem MH, Rost C, Klinghammer L, Hennig FF, Daniel WG, et al. Early repolarization, left ventricular diastolic function, and left atrial size in professional soccer players. $\mathrm{Am} \mathrm{J}$ Cardiol 2010; 106: 569-574.

9. Haïssaguerre M, Shoda M, Jaïs P, Nogami A, Shah DC, Kautzner $\mathrm{J}$, et al. Mapping and ablation of idiopathic ventricular fibrillation. Circulation 2002; 106: 962-967.

10. Noda T, Shimizu W, Taguchi A, Aiba T, Satomi K, Suyama K, et al. Malignant entity of idiopathic ventricular fibrillation and polymorphic ventricular tachycardia initiated by premature extrasystoles originating from the right ventricular outflow tract. $J \mathrm{Am}$ Coll Cardiol 2005; 46: 1288-1294.

11. Van Herendael H, Zado ES, Haqqani H, Tschabrunn CM, Callans DJ, Frankel DS, et al. Catheter ablation of ventricular fibrillation:
Importance of left ventricular outflow tract and papillary muscle triggers. Heart Rhythm 2014; 11: 566-573.

12. Saliba W, Abul Karim A, Tchou P, Natale A. Ventricular fibrillation: Ablation of a trigger? J Cardiovasc Electrophysiol 2002; 13: $1296-1299$.

13. Anter E, Buxton AE, Silverstein JR, Josephson M. Idiopathic ventricular fibrillation originating from the moderator band. $J$ Cardiovasc Electrophysiol 2013; 24: $97-100$.

14. Klatsky AL, Oehm R, Cooper RA, Udaltsova N, Armstrong MA. The early repolarization normal variant electrocardiogram: Correlates and consequences. Am J Med 2003; 115: 171-177.

15. Haïssaguerre M, Sacher F, Nogami A, Komiya N, Bernard A, Probst V, et al. Characteristics of recurrent ventricular fibrillation associated with inferolateral earlyrepolarization role of drug therapy. J Am Coll Cardiol 2009; 53: 612-619.

16. Antzelevitch C, Yan GX. J-wave syndromes. Heart Rhythm 2010; 7: $549-558$

17. Takeuchi T, Sato N, Kawamura Y, Takahashi F, Sato M, Kikuchi $\mathrm{K}$, et al. A case of a short-coupled variant of torsades de pointes with electrical storm. Pacing Clin Electrophysiol 2003; 26: $632-$ 636.

18. Haïssaguerre M, Shah DC, Jaï P, Shoda M, Kautzner J, Arentz $\mathrm{T}$, et al. Role of Purkinje conducting system in triggering of idiopathic ventricular fibrillation. Lancet 2002; 359: 677-678.

19. Antzelevitch C, Viskin S. Brugada Syndrome: Cellular mechanisms and approaches to therapy. In: Gussak I, Antzelevitch C, editors. Electrical diseases of the heart: Genetics, mechanisms, treatment, prevention. London: Springer-Verlag, 2008; 500-535.

20. Nam GB, Ko KH, Kim J, Park KM, Rhee KS, Choi KJ, et al. Mode of onset of ventricular fibrillation in patients with early repolarization pattern vs. Brugada syndrome. Eur Heart J 2010; 31: $330-339$.

21. Nakagawa M, Ezaki K, Miyazaki H, Wakisaka O, Shinohara T, Teshima Y, et al. Takahashi N, Saikawa T. Electrocardiographic characteristics of patients with false tendon: Possible association of false tendon with J waves. Heart Rhythm 2012; 9: 782-788.

\section{Supplementary Files}

Please find supplementary file(s);

http://dx.doi.org/10.1253/circj.CJ-19-0021 